\title{
Detection of R-Peaks in ECG Signal by Adaptive Linear Neuron (ADALINE) Artificial Neural Network
}

\author{
Jeong-Hwan Kim, Sang-Eun Park, Gyeo-Wun Jeung, Kyeong-Seop Kim \\ School of Biomedical Engineering, Konkuk University, KOREA
}

\begin{abstract}
This research proposes a new method to detect R-peaks in electrocardiogram by using the prediction value from adaptive linear neuron (ADALINE) artificial neural network. With this aim, the weights of four input neurons in ADALINE are updated for each encoded ECG vector-segment and the value of an output neuron is compared with the actual ECG followed by applying finite impulse response filter. Our simulated experiments with the MIT-BIH ECG database that represents the long-term recordings from the heart disease patients show that our proposed algorithm can detect R-peaks in ECG data with the accuracy of more than $99 \%$.
\end{abstract}

Keywords: ECG, R-peak, neural network, ADALINE, arrhythmia, Premature Ventricular Contraction, finite impulse response filter, MIT-BIT database.

\section{Introduction}

Electrocardiogram (ECG) that records the electric potentials resulting from a heart's activity can offer the clinical decision parameters to diagnose an abnormal heart sinus rhythm. The primary features that characterize ECG waveform include R-peak, QRS complex, S and T fiducial points in terms of time-location and morphological shape [1].

In the ECG data, the most crucial information is the time locations occurring in R-peak because this feature can be utilized to monitor especially Premature Ventricular Contraction (PVC) arrhythmia [2] and Heart Rate Variability (HRV) [3] in the long-term based heart health monitoring environment. So far, concerning the automatic detection of R-peaks in ECG data, the adaptive threshold level was sought [4] or a differential operator was applied to compute the gradient of ECG waveform [5]. Also R-peak characteristic point was estimated by encoding the ECG data in wavelet transform $[6,7,8]$ or Hilbert transform domain [9]. In our study, a new algorithm to detect R-peaks is proposed by evaluating the predicting feature from ADALINE artificial neural network model $[10,11]$ and comparing with the actual ECG voltage level. With this aim, ECG data is encoded successively in a four dimensional vector-segment format and feed forwards into four input neurons in ADALINE to update their weights. The value of an output neuron is computed as a prediction value for each ECG segment and compared with the actual voltage level to estimate the time locations of R-peak.
2.1 ADALINE artificial neural network architecture ADALINE has single output neuron that receives the weighted sum from the multiple input neurons with an optional bias. Fig. 1 shows the architecture of an ADALINE neural net consisted with $n$-input neurons $X_{l}$, $X_{2}, \ldots, X_{n}$, an output neuron $y$ with specifying weights of $w_{1}, w_{2}, \ldots, w_{n}$ and a bias $b$.

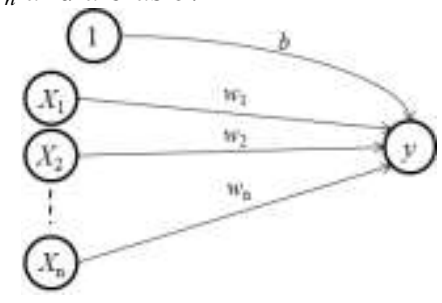

Figure 1. Architecture of an ADALINE neural net.

In the ADALINE model, the weights of connections between input neurons and an output neuron are trained using the delta rule [12]. Training and testing algorithm to extract the residual error between the predicted ECG and the real data as following steps;

Step 1: Initialize weights $w_{1}, w_{2}, w_{3}, w_{4}$ and set learning rate $\alpha=10^{-6}$.

Step 2: Encode ECG input data $X=\left[X_{1}, X_{2}, X_{3}, \ldots\right.$, $\left.X_{N}\right], N$ : the number of samples, into an $i^{\text {th }}$ segment $\boldsymbol{S}_{i}, i=$ $1,2, \ldots . N$ in a fourth dimensional vector format:

$\boldsymbol{S}_{1}=\left[\begin{array}{c}x_{1} \\ 0 \\ 0 \\ 0\end{array}\right], \boldsymbol{S}_{2}=\left[\begin{array}{c}x_{2} \\ x_{1} \\ 0 \\ 0\end{array}\right], \boldsymbol{S}_{2}=\left[\begin{array}{c}x_{3} \\ x_{2} \\ x_{1} \\ 0\end{array}\right], \cdots, \boldsymbol{S}_{N-1}=\left[\begin{array}{c}x_{N-1} \\ x_{N-2} \\ x_{N-3} \\ x_{N-4}\end{array}\right], \boldsymbol{S}_{N}=\left[\begin{array}{c}x_{N} \\ x_{N-1} \\ x_{N-2} \\ x_{N-3}\end{array}\right]$

\section{Prediction of ECG data by adaline neural network}


Step 3: Compute $i^{\text {th }}$ predicted vale from an output neuron by activating $(i-1)^{t h}$ weights:

$$
y_{i}=b+\boldsymbol{S}_{i} \cdot \boldsymbol{W}^{i-1}
$$

, where $\cdot$ is a dot product operator and $\boldsymbol{W}^{i-1}=$ $\left[w_{1}^{i-1}, w_{2}^{i-1}, w_{3}^{i-1}, w_{4}^{i-1}\right]^{t}$ ( $t$ : transpose) is a weight vector that was determined at $(i-1)^{\text {th }}$ training stage

Step 4: Update and weights, $i=1,2,3$, 4, with nullifying a bias effect (i.e., $b=0$ ):

$\boldsymbol{W}^{i}=\boldsymbol{W}^{i-1}+\alpha \cdot\left(t_{i}-y_{i}\right) \cdot x_{i}, t_{i}$ : the target ECG data

During the process of training algorithm, the weights are updated adaptively for each feed forwards ECG segment $\boldsymbol{S}_{i}$ so as to minimize the residual error between the real ECG data $X_{i}$ and the output vale $y_{i}$. In order to evaluate the performance in detecting R-peaks in ECG data, we use the MIT-BIH database [13] which recorded the long-term ECG readings with the sampling rate of $360 \mathrm{~Hz}(180 \mathrm{~Hz}$ in text file format) from the heart disease patients. For our experiments, the record titled with ECG100_1 (contains the normal sinus rhythms and one PVC beat) and ECG119_1 (includes a quite number of PVC abnormal heart beats) are considered. As a preprocessing step, a digital high-pass finite impulse response (FIR) filter is initially applied to the considered ECG data to eliminate the baseline wandering noise [14].

\section{2 ADALINE artificial neural network architecture}

Fig.2-(a) shows that the part of residual error $e_{i}, i=1, \ldots$, $N$, between the predictive value from an output neuron as an estimate of ECG data $x_{i}$ (ECG100_1) and the real data. Fig.2-(b) describes the updating weights when $i^{\text {th }}$ ECG segment $\boldsymbol{S}_{i}$ (ECG100_1) feeds into the input neurons. In this figure, the error signal is abruptly changed in the neighborhood area of R-peak. Thus we can utilize this fact that the initial R-peaks candidates can be selected with applying a moving average filter followed by lowpass FIR digital filter to smooth the residual peaks and to set the adaptive threshold line in order to delineate Rpeak candidates.

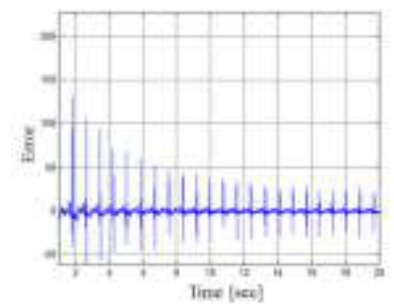

(a)

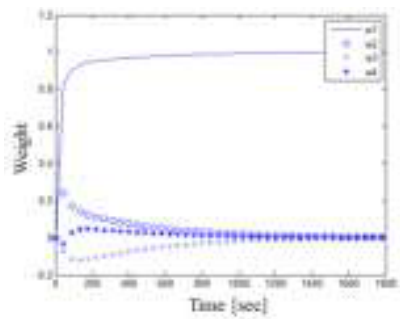

(b)
Figure 2. (a) The part of residual error $e^{i}$ for ECG100 1 dataset. (b) Updating weights of ADALINE neural net for ECG100_dataset.

\section{Detection of R-peaks in ECG signal}

In order to smooth the sharpness in the residual peaks, the moving average filter (window size, $L$ is selected to be 20) is applied and eps (error in prediction signal from ADALINE) is defined as in equation (4).

$$
e^{e p s^{i}}=\left(\frac{1}{L}\right) \cdot \sum_{j=0}^{L-1}\left|e^{j}\right|, i=1,2, \ldots, N
$$

To set the threshold line that is adaptively tune to the trend of residual error signal, eps is low-pass filtered by a FIR digital filter (see Table 1 for filter design specification). As of a result, Fig. 3 shows eps ${ }^{i}$ with lowpass filtered eps signal, leps ${ }^{i}, i=1, \ldots, N$. Here leps ${ }^{i}$ is used as a reference level to delineate the time intervals including R-peaks.

Table 1. Design specifications of low-pass FIR filter for extracting leps ${ }^{i}$

\begin{tabular}{cc}
\hline \hline Parameters & Value \\
\hline Window function & Hanning \\
Sampling freq. & $180 \mathrm{~Hz}$ \\
Filter order & 599 \\
Pass band cutoff freq. & $0.5 \mathrm{~Hz}$ \\
Stop band cutoff freq. & $1.5 \mathrm{~Hz}$ \\
\hline \hline
\end{tabular}
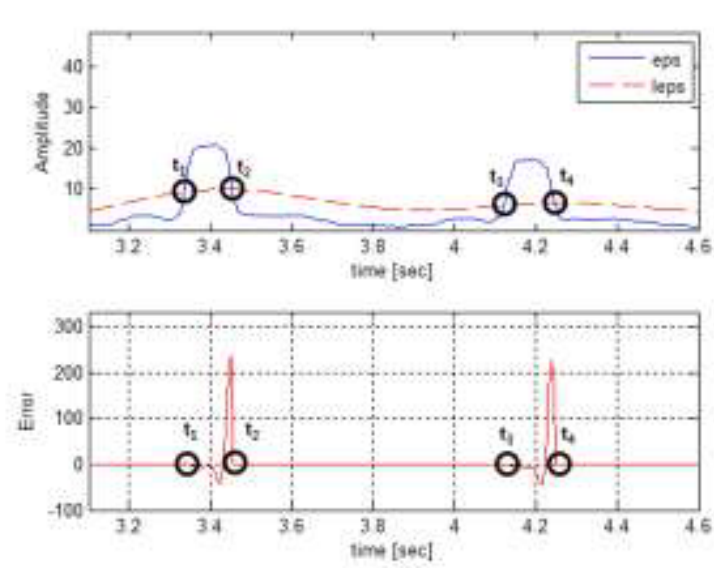

Figure 3. The illustration of residual error: eps ${ }^{i}$ (solid line), leps $^{i}$ (dotted line) in the top chart and the overall trend of leps ${ }^{i}$ in the below one.

Finally, the time locations in R-peak can be resolved by computing the gradient value for each leps-segment for $t_{i} \leq t \leq t_{j}$. Fig.4 illustrates the result of R-peak detection as a symbol of 'o' for ECG100 1 and for ECG119_1. Note that two R-peaks in ECG 119 1 are missed especially during the initial weights updating stage (i.e., the time less than 2 seconds) due to the inherent characteristics of neural network model. In other words, ADALINE needs some warming up time to trace the trend of input ECG data $\boldsymbol{S}_{i}$. The accuracy of estimating R-peaks by ADALINE can be slightly varied upon the size of moving average window $L$. Nonetheless, the overall performance of detecting R-peaks by ADALINE is superior to the conventional method that simply computes the gradient of ECG waveform. Table 2 and 3 summarize the performance from ADALINE and differential operator in terms of TP (True Positive), TN (True Negative), FP (False Positive) and FN (False Negative) in detecting R-peaks for ECG100_1 and 
ECG119_1, respectively. In Table 2, Sensitivity and Accuracy are defined as follows [15];

$$
\begin{gathered}
\text { Sensitivity }=\frac{\mathrm{TP}}{\mathrm{TP}+\mathrm{FN}} \times 100(\%), \\
\text { Accuracy }=\frac{\mathrm{TP}+\mathrm{TN}}{\mathrm{TP}+\mathrm{FP}+\mathrm{FN}+\mathrm{TN}} \times 100(\%)
\end{gathered}
$$

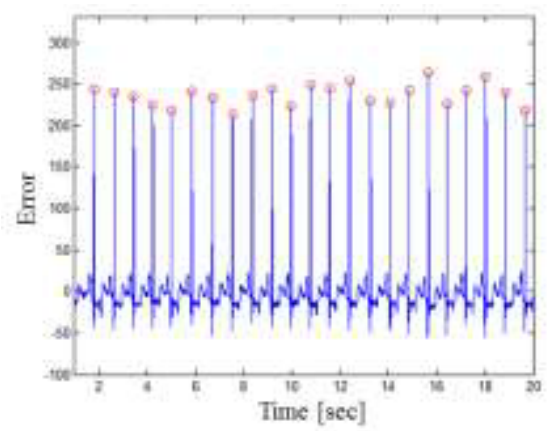

(a)

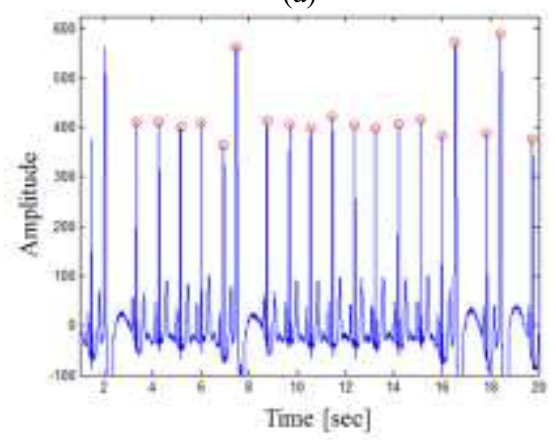

(b)

Figure 4. The results of R-peak detection as a symbol of ' $o$ ': (a) ECG100_1, $L=20$ (b) ECG119_1, $L=20$.

Table 2. Comparison of performance in detecting R-peaks by ADALINE using the different size of window $L$ with applying differential operator to ECG100_1

\begin{tabular}{ccccccc}
\hline \hline & TP & TN & FP & FN & $\begin{array}{c}\text { Sensitivity } \\
(\%)\end{array}$ & $\begin{array}{c}\text { Accuracy } \\
(\%)\end{array}$ \\
\hline $\begin{array}{c}\text { Differential } \\
\text { operator[5] }\end{array}$ & 2,151 & 0 & 10 & 99 & 95.6 & 95.18 \\
$\begin{array}{c}\text { ADALINE } \\
(L=10)\end{array}$ & 2,258 & 0 & 1 & 0 & 100 & 99.96 \\
$\begin{array}{c}\text { ADALINE } \\
(L=15)\end{array}$ & 2,256 & 0 & 2 & 1 & 99.96 & 99.87 \\
$\begin{array}{c}\text { ADALINE } \\
(L=20)\end{array}$ & 2,256 & 0 & 1 & 2 & 99.91 & 99.87 \\
\hline \hline
\end{tabular}

Table 3. Comparison of performance in detecting Rpeaks by ADALINE with applying the differential operator to ECG119_1 dataset

\begin{tabular}{ccccccc}
\hline \hline & TP & TN & FP & FN & $\begin{array}{c}\text { Sensitivity } \\
(\%)\end{array}$ & $\begin{array}{c}\text { Accuracy } \\
(\%)\end{array}$ \\
\hline $\begin{array}{c}\text { Differential } \\
\text { operator[5] }\end{array}$ & 1,343 & 0 & 365 & 220 & 85.92 & 69.67 \\
ADALINE & 1,974 & 0 & 8 & 2 & 99.9 & 99.5
\end{tabular}

$(L=10)$

\begin{tabular}{ccccccc}
$\begin{array}{c}\text { ADALINE } \\
(L=15)\end{array}$ & 1,975 & 0 & 0 & 2 & 99.9 & 99.9 \\
$\begin{array}{c}\text { ADALINE } \\
(L=20)\end{array}$ & 1,972 & 0 & 0 & 2 & 99.9 & 99.9 \\
\hline \hline
\end{tabular}

In summarizing our proposed algorithm, ADALINE neural net accompanied with the necessary digital filtering processes is described in Fig.5.

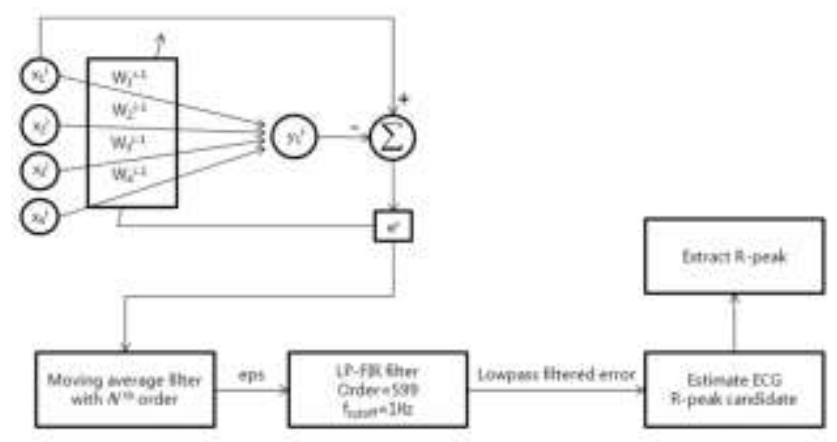

Figure 5. The proposed ADALINE neural net for detecting R-peaks in the ECG data.

\section{Conclusion}

In this research, we can find the fact that R-peaks in ECG can be automatically detected with the high accuracy of more than $99 \%$ by ADALINE neural net implemented with four input neurons and one output neuron. Concerning the clinical decisions for the diagnosis of arrhythmia, FN index is the most crucial feature. As shown in Table 2 and 3, the number of FN's are $2(L=20)$ for ECG 100_1 and ECG 119_1 dataset, respectively. The main cause of this misclassification is the limited capacity of ADALINE neural network model when it deals with updating weights especially during the initial training stage.

\section{Acknowledgment}

This research was supported by Basic Science Research Program through the National Research Foundation of Korea (NRF) funded by the Ministry of Education, Science and Technology (NRF-2013R1A1A2012393).

\section{References}

1. A. U. Rajendra, J. S. Suri, J. A. E. Spaan and S. M. Krishnan, Advances in Cardiac Signal Processing, Berlin Heidelberg: Springer, (2007).

2. M. L. Talb and A. Charef, "PVC discrimination using the QRS power spectrum and self-organizing meps," Computer Methods and Programs in Biomedicine, 94, 223-231, (2009).

3. M. V. Kamath, M. A. Watanabe and A. R. M. Upton, Heart Rate Variability (HRV) Signal Analysis: Clinical Applications. CRC Press, (2013).

4. F. Chiarugi, V. Sakkalis, D. Emmanouilidou, T. Krontiris, M. Varanini and I. Tollis, "Adaptive 
threshold QRS detector with best channel selection based on a noise rating system," IEEE Computers in Cardiology, 157-160, (2007).

5. J. Pan and W. J. Tompkins, "A real-time QRS detection algorithm," IEEE Transactions on Biomedical Engineering, 32, 230-236, (1985).

6. C. Li, C. Zheng and C. Tai, "Detection of ECG characteristic points using wavelet transforms," IEEE Transactions on Biomedical Engineering, 42, 21-28, (1995).

7. P. J. M. Fard, M. H. Moradi and M. R. Tajvidi, "A novel approach in $\mathrm{R}$ peak detection using Hybrid Complex Wavelet (HCW)," International Journal of Cardiology, 124, 250-253, (2008).

8. C. Li, C. Zheng and C. Tai, "Detection of ECG characteristic points using wavelet transforms," IEEE Transactions on Biomedical Engineering, 22, 21-28, (1995).

9. D. S. Benites, P. A. Gaydeck, A. Zaidi and A. P. Fitzpatrick, "A new QRS detection algorithm based on the Hilbert transform," IEEE Computers in Cardiology, 379-382, (2000).

10. L. Fausett, Fundamentals of Neural Networks, Upper Saddle River: Prentice Hall, (1994).

11. S. N. Sivanandam, S. Sumathi and S. N. Deepa, Introduction to neural networks using Matlab 6.0, McGraw-Hill Education, (2006).

12. Y. H. Hu and J. N. Hwang, Handbook of Neural Signal Processing, CRC Press, (2002).

13. A. L. Goldberger, "PhysioBank, PhysioToolkit, and Physionet: components of a new research resource for complex physiologic signals," Circulation, 23, 215-220, (2000).

14. J. H. Kim, S. E. Park, J. W. Lee and K. S. Kim, "Design and implementation of digital filters for mobile healthcare applications," International Journal of Electronics and Electrical Engineering, 2, 75-79, (2014).

15. W. L. Martinez, Computational Statistics Heandbook with Matlab, Chapman \& Hall, CRC Press, (2008). 